\title{
HARDWARE-IN-THE-LOOP TESTS AND ANALYSIS OF HVDC SYSTEM'S IMPACT ON DISTANCE PROTECTION PERFORMANCE Di Liu ${ }^{1}$, Qiteng Hong ${ }^{1 *}$, Adam Dyśko ${ }^{1}$, Dimitrios Tzelepis ${ }^{1}$, Campbell Booth ${ }^{1}$, Ian Cowan ${ }^{2}$, and Bharath Ponnalagan ${ }^{2}$
}

\author{
${ }^{1}$ Department of Electronic and Electrical Engineering, University of Strathclyde, Glasgow, United Kingdom \\ ${ }^{2}$ The National HVDC Centre, Glasgow, United Kingdom \\ *E-mail: q.hong@strath.ac.uk
}

\begin{abstract}
Keywords: DISTANCE PROTECTION, HVDC SYSTEM, REAL TIME DIGITAL SIMULATION, HARDWARE-IN-THE-LOOP TESTS.
\end{abstract}

\begin{abstract}
This paper presents comprehensive and realistic Hardware-In-the-Loop (HIL) tests of a physical relay and analysis of the test results for evaluating the impact of HVDC systems (and converters in general) on the operation of distance protection. In the established HIL test configuration, simulated voltage and current waveforms from a Real Time Digital Simulator (RTDS) are injected to the relays via an analogue amplifier, and the relays' tripping signals are input back to the RTDS to monitor their tripping actions. During the HIL tests, the relay is configured with both MHO and QUAD characteristics, and it is tested under a wide range of system operating conditions with different fault levels, fault types and locations, and HVDC control strategies. The test results show that the integration of the HVDC system could lead to the compromised distance protection performance, including failed tripping, delayed tripping and zone discrimination issues. Detailed analysis of the test results is presented, and it is found that the main causes of the identified issues include: 1) under-reach/over-reach problem owing to the angle difference of currents from local and remote ends in the event of resistive faults; 2) inaccurate impedance measurement problem due to identical faulty phase currents during phase-to-phase faults with the constant reactive power control of HVDC system; 3) phase selection issues owing to the abnormal increase of the superimposed currents during phase-earth fault with balanced current control (i.e., only injecting positive sequence current without any negative sequence component) of HVDC system. The results and analysis presented in this paper will not only offer valuable evidence-based insights to understand the challenges of distance protection in future converter-dominated networks, but also provide a useful reference, informing future research and development to address these identified issues.
\end{abstract}

\section{Introduction}

The GB transmission system is expected to see a massive increase of HVDC capacity and Non-Synchronous Generation (NSG) in the coming decades to achieve the ambitious net-zero carbon emission target by 2050 [1]. HVDC systems and NSG units (e.g. wind and solar generation) are both interfaced with the AC power networks via power electronic converters, which have significantly different fault characteristics compared with conventional Synchronous Generators (SGs), and their fault responses are largely governed by the embedded controllers [2]. Furthermore, due to the limited overcurrent capability of semiconductor devices, the fault current contributed by the converters is much smaller compared with SGs and is typically within $1.5 \mathrm{pu}$ [3]. Therefore, the massive integration of HVDC systems, and in general converter based NSG, presents significant challenges for protection systems.

Distance protection plays a critical role in safeguarding transmission networks during faults, which operates by comparing the measured impedance with the preconfigured zone reaches. In [4], HIL tests are conducted to investigate the HVDC system's impacts on distance protection performance. It was found that after the connection of a HVDC system, the distance relay could potentially trip in the false zone and with a longer delay (> $140 \mathrm{~ms}$ ), particularly in the grid with low fault level. However, this paper does not conduct detailed theoretical analysis to explain the fundamentals of those observed issues. As reported in [5][6], owing to the different fault responses of converters from SGs, there will exist a large angle difference between the fault currents from the local and remote ends of the protected line, which can result in an inaccurate measurement of the faulted loop impedance, and subsequently lead to the over/under-reach issues of distance protection. The impact of various control strategies on the distance protection (e.g., balanced current control, constant active power control and constant reactive power control) has been considered in [7]. It suggests that the constant reactive power controller [8] should be avoided from the protection aspect as it can lead to the computation issues in phase to phase impedance measuring elements of the distance relay. However, these papers only focus on the accurate impedance measurement, without considering the other critical protection elements such as the starting unit, faulty phase selection unit, etc. Furthermore, the impact of different relay characteristics (e.g., MHO and QUAD) have not been considered. In this paper, HIL tests of a physical relay 


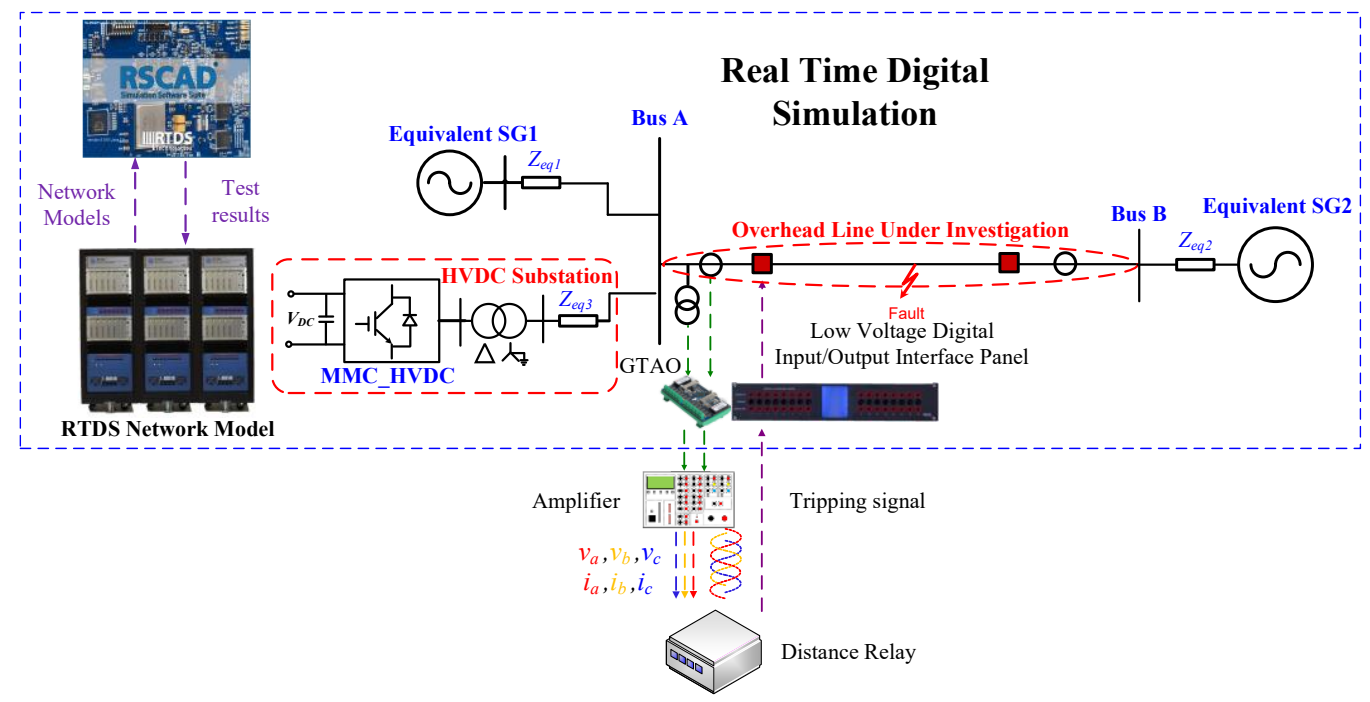

Fig. 1. Schematic of the studied network and the HIL arrangement.

will be presented, along with the theoretical analysis to investigate the potential protection issues of the distance relay due to the integration of HVDC systems (and converters in general). Multiple factors, including different fault conditions, protection characteristics, levels of system strength and HVDC control modes, are all considered in this study.

The paper is structured as follows: Section 2 provides an overview of the tested network and the setup of the developed HIL test platform; Section 3 presents the mathematic analysis of the faulted loop impedance and analyses the under/over reach issues of distance protection; Section 4 presents studied cases and analyses the tested results; Section 5 provides the conclusions of this paper.

\section{Overview of the Studied Network and HIL Test Setup}

\subsection{Modelling of the studied network}

The studied network and the setup of the HIL testing platform are shown in Fig. 1. As presented in this figure, SG1 and SG2 are the voltage sources connected to the protected line, which represent the equivalent "lumped" synchronous generation sources at the busbars; $Z_{e q 1}$ and $Z_{e q 2}$ are the equivalent impedances of SG1 and SG2, which directly determine the fault levels at the Bus $\mathrm{A}$ and $\mathrm{B} ; Z_{e q 3}$ represent the equivalent impedance of the HVDC unit with the grid, which can be manually adjustable by the users to ensure the operating stability of the HVDC unit; the MMC-HVDC represents the Modular Multi-Level Converter (MMC) interfaced HVDC system, where a flexible controller with the current references described in (1) to (4) has been designed to realise different control strategies (e.g., balanced current control, constant active power control and constant reactive power control) and inject the positive sequence reactive current during faults, following the requirements specified in the GB Grid Code [9], to support the depressed voltage during faults.

$$
i_{d r}^{+}=\sqrt{1.2^{2}-\left(i_{q r}^{+}\right)^{2}}
$$

$$
\begin{gathered}
i_{q r}^{+}=-3.28 v^{+}+2.64,0 \leq i_{q r}^{+} \leq 1 \\
i_{d r}^{-}=K_{\text {mode }}\left(\frac{v_{d}^{+} v_{d}^{-}-v_{q}^{+} v_{q}^{-}}{v_{d}^{+2}+v_{q}^{+2}}\right) i_{d r}^{+}+K_{\text {mode }}\left(\frac{v_{d}^{+} v_{q}^{-}+v_{d}^{-} v_{q}^{+}}{v_{d}^{+2}+v_{q}^{+2}}\right) i_{q r}^{+} \\
i_{q r}^{-}=K_{\text {mode }}\left(\frac{v_{d}^{+} v_{q}^{-}+v_{d}^{-} v_{q}^{+}}{v_{d}^{+2}+v_{q}^{+2}}\right) i_{d r}^{+}+K_{\text {mode }}\left(\frac{v_{q}^{+} v_{q}^{-}-v_{d}^{+} v_{d}^{-}}{v_{d}^{+2}+v_{q}^{+2}}\right) i_{q r}^{+}
\end{gathered}
$$

where $i_{d r}^{+}, i_{q r}^{+}, i_{d r}^{-}$and $i_{q r}^{-}$are the positive and negative sequence current references in the $d q$ frame; $v_{d}^{+}, v_{q}^{+}, v_{d}^{-}$and $v_{q}^{-}$are the positive and negative sequence voltage references in the $d q$ frame; $K_{\text {mode }}$ is a variable defined to control the operating modes of the HVDC system, which can operate with the constant reactive power controller, balanced current controller and constant active power controller when the values of $K_{\text {mode }}$ are set as 1,0 and -1 respectively.

The current contributed by the proposed MMC-HVDC unit is limited to $1.2 \mathrm{pu}$ to avoid the overcurrent issues and the distance relay installed at the HVDC side is investigated as indicated in Fig. 1. Detailed information about the network development can be found in the modelling report of the project in [10].

\subsection{Arrangement of the HIL testing platform}

As illustrated in Fig. 1, the network model is developed in RSCAD (a software package specially designed for RTDS) and run in real time in the RTDS simulator. The secondary side three-phase voltage and current waveforms at the relay point are measured by the voltage and current transformer (VT and CT) models [11] available in the RSCAD library, which are further scaled down so that they fall into the output voltage range of the analogue output GTAO card (i.e. ${ }_{-}^{+} 10 \mathrm{~V}$ ). The output signals from the GTAO card are amplified to the same voltage and current levels as the outputs of the VT and CT using an analogue amplifier. The amplified voltage and current are then injected to the physical relay, which makes decisions on the tripping actions based on the input voltage and current. If the relay trips, the tripping signal will be transmitted back to the RTDS simulator and recorded in the RSCAD. 


\section{Theoretical Analysis of Distance Protection Performance}

\subsection{Impedance measured by distance relay}

The network shown in Fig. 1 is used to illustrate the derivation of the faulted loop impedance measured by the distance relay. For the faults at the protected line, the impedance of distance relay is depicted in (5), which can be derived by applying the Kirchhoff Voltage Law (KVL) from the faulted point to the relay installation point.

$$
Z_{M}=\frac{V_{M}}{I_{M}}=\frac{I_{L} \times Z_{L}+\left(I_{L}+I_{R}\right) R_{F}}{I_{L}}=Z_{L}+\left(1+\frac{I_{R}}{I_{L}}\right) R_{F}=Z_{L}+Z_{E}
$$

where $Z_{M}$ is the impedance measured by the relay; $V_{M}$ and $I_{M}$ are the relay measured voltage and current; $I_{L}$ and $I_{R}$ are the current infeed from the local (Bus A) and remote ends (Bus B) of the protected line; $Z_{L}$ is the impedance of the line between the fault and relay location; $Z_{E}$ is the error of the measured impedance; $R_{F}$ is the fault resistance. The representation of $I_{L}$ and $I_{R}$ in (5) referring to the different impedance measuring elements of distance relay are displayed in Table 1 , where the subscripts $A, B$ and $C$ refer to phases $\mathrm{A}, \mathrm{B}$ and $\mathrm{C}$; $L$ and $R$ refer to the local and remote ends; $K_{0}$ is the residual compensation factor and $I_{g}$ is the grounding current of relay.

Table 1 Representation of $I_{L}$ and $I_{R}$ of all impedance elements

\begin{tabular}{ccc}
\hline Impedance Elements & $I_{L}$ & $I_{R}$ \\
\hline $\mathrm{AG}$ & $I_{A}^{L}+K_{0} I_{g}$ & $I_{A}^{R}-K_{0} I_{g}$ \\
$\mathrm{BG}$ & $I_{B}^{L}+K_{0} I_{g}$ & $I_{B}^{R}-K_{0} I_{g}$ \\
$\mathrm{CG}$ & $I_{C}^{L}+K_{0} I_{g}$ & $I_{C}^{R}-K_{0} I_{g}$ \\
$\mathrm{AB}$ & $I_{A}^{L}-I_{B}^{L}$ & $I_{A}^{R}-I_{B}^{R}$ \\
$\mathrm{BC}$ & $I_{B}^{L}-I_{C}^{L}$ & $I_{B}^{R}-I_{C}^{R}$ \\
$\mathrm{CA}$ & $I_{C}^{L}-I_{A}^{L}$ & $I_{C}^{R}-I_{A}^{R}$ \\
\hline
\end{tabular}

\subsection{Under/Over-reach issues of distance protection}

As revealed by (5), an error impedance, $Z_{E}$, is introduced to the measured impedance of distance relay when the value of $R_{F}$ is not zero. For the network dominated by the SGs, the phase angles of $I_{R} / I_{L}$ (represented as $\Delta \psi$ in the following discussion) are typically small, thus the increase or decrease of the imaginary part of the error impedance is generally negligible [12]. Therefore, the distance relay is largely immune to the under-reach or over-reach issues as shown in Fig. 2 (a). However, the above scenario can change significantly when the fault infeed from SG1 at Bus A is low and an HVDC system (and in general converters) is connected, leading to the fault current $I_{L}$ mainly contributed by the converter, thus its characteristics are highly dependent on the converter control algorithm.

In the existing HVDC systems, the balanced current control is one of the most commonly used control strategies, so it is used as the example to illustrate the fundamentals of the over-reach and under-reach issues of distance protection with integration of HVDC systems. The fault current contributed by the HVDC system with balanced current controller is depicted in (6) [13].

$$
i_{P}(t)=\sqrt{i_{d}^{+2}+i_{q}^{+2}} \sin \left(\omega t+\arctan \left(\frac{i_{q}^{+}}{i_{d}^{+}}\right)+\theta_{v d}^{+}+\theta_{P}\right)
$$

where $i_{P}$ is the phase current injected by the HVDC system and $P$ refers to phase $\mathrm{A}, \mathrm{B}$ and $\mathrm{C} ; i_{d}^{+}$and $i_{q}^{+}$are the injected positive sequence currents in the $d q$ axes; $\omega$ is the system angular frequency; $\theta_{v d}^{+}$is the initial angle of the positive sequence voltage in the $d$ axes; $\theta_{P}$ is the phase angle, i.e. $0^{\circ}$, $-120^{\circ}$ and $120^{\circ}$ for phase $\mathrm{A}, \mathrm{B}$ and $\mathrm{C}$ respectively. According to (6), the angle of phase current injected by the HVDC unit can vary between $0^{\circ}$ to $360^{\circ}$ (subject to the control strategy being deployed), which leads to the angle of $I_{R} / I_{L}, \Delta \psi$, in (5) ranges from $0^{\circ}$ to $360^{\circ}$ as well. When the value of $\Delta \psi$ belongs to $0^{\circ}$ to $180^{\circ}$, the under-reach issues will be observed in the distance protection as shown in Fig. 2 (b). The over-reach issues displayed in Fig. 2 (c) will be introduced for the $\Delta \psi$ ranging from $180^{\circ}$ and $360^{\circ}$ [13].

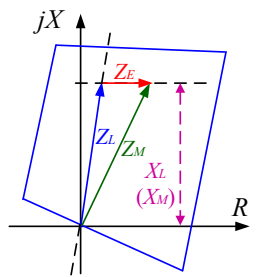

(a)

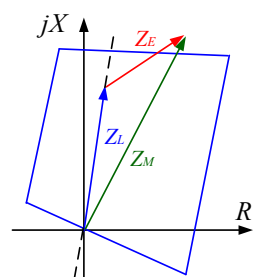

(b)

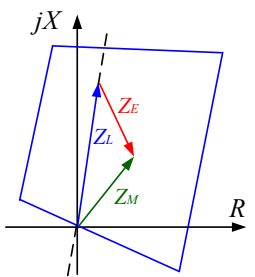

(c)
Fig. 2 Measured impedance of distance relay with (a) $\Delta \psi=$ $0^{\circ}$, (b) $\Delta \psi \in\left(0^{\circ}, 180^{\circ}\right)$, (c) $\Delta \psi \in\left(180^{\circ}, 360^{\circ}\right)$

\section{Case Studies - HIL Tests}

\subsection{Information of the studied cases and relay settings}

The parameters of the protected line as illustrated in Fig. 1 are listed in Table 2 and the information of the studied cases is shown in Table 3, where $F L_{S G 1}$ and $F L_{S G 2}$ are the fault infeeds from SG1 and SG2 respectively; $\mathrm{BI}, \mathrm{CP}$ and $\mathrm{CQ}$ refer to the balanced current control [14], constant active power control [15] and constant reactive power control [8]. In Table 3, case 1 and case 2 are used as the reference cases, where the network only contains SGs. Case 3 to case 5 are used to investigate the impact of HVDC control modes on the distance protection performance, where the fault infeed from SG1 is set as 0 MVA. Based on the test results of case 3 to case 5, the relay fails to trip when the HVDC system uses the balanced current control (case 3). Therefore, case 6 is designed, where the SG1 with 839 MVA fault level (same as the HVDC capacity), to evaluate the impacts of synchronous compensation. In case 7 and $8, \mathrm{AB}$ faults are applied and the fault level of SG1 are set as 0 MVA. In case 9 and case 10, actual system fault levels of two substations in Scotland (i.e. the Spittal and the remotely connected substation) are implemented to test the distance protection performance and the relay performance with three phase faults are studied in case 11 and 12.

Table 2 Parameters of the protected line

\begin{tabular}{ccc}
\hline Parameter & Defination & Values \\
\hline$L$ & Length of the protected line & $12.1 \mathrm{~km}$ \\
& Positive and zero sequence & $r_{1}=0.0378 \Omega / \mathrm{km}$ \\
$r_{1}, r_{0}$ & resistance per km & $r_{0}=0.159 \Omega / \mathrm{km}$ \\
& Positive and zero sequence & $l_{1}=1.324 \mathrm{mH} / \mathrm{km}$ \\
$l_{1}, l_{0}$ & inductance per km & $l_{0}=3.202 \mathrm{mH} / \mathrm{km}$ \\
& Positive and zero sequence & $c_{1}=8.964 \mathrm{nF} / \mathrm{km}$ \\
$c_{1}, c_{0}$ & capacitance per km & $c_{0}=6.48 \mathrm{nF} / \mathrm{km}$ \\
\hline
\end{tabular}


Table 3 Information of the studied cases

\begin{tabular}{|c|c|c|c|c|}
\hline Cases & $F L_{S G 1}$ & $F L_{S G 2}$ & HVDC Control & Fault Condition \\
\hline 1 & 839 MVA & 3000 MVA & N/A & $\mathrm{AG}, 15 \%, 2 \Omega$ \\
\hline 2 & 839 MVA & 3000 MVA & N/A & $\mathrm{AB}, 15 \%, 2 \Omega$ \\
\hline 3 & 0 MVA & 3000 MVA & BI & $\mathrm{AG}, 15 \%, 2 \Omega$ \\
\hline 4 & $0 \mathrm{MVA}$ & 3000 MVA & $\mathrm{CP}$ & $\mathrm{AG}, 15 \%, 2 \Omega$ \\
\hline 5 & $0 \mathrm{MVA}$ & 3000 MVA & CQ & $\mathrm{AG}, 15 \%, 2 \Omega$ \\
\hline 6 & 839 MVA & 3000 MVA & $\mathrm{BI}$ & $\mathrm{AG}, 15 \%, 2 \Omega$ \\
\hline 7 & 0 MVA & 3000 MVA & $\mathrm{BI}$ & $\mathrm{AB}, 15 \%, 2 \Omega$ \\
\hline 8 & 0 MVA & 3000 MVA & CQ & $\mathrm{AB}, 15 \%, 1 \mathrm{e}-9 \Omega$ \\
\hline 9 & 1836 MVA & 1372 MVA & $\mathrm{BI}$ & $\mathrm{AG}, 15 \%, 2 \Omega$ \\
\hline 10 & 1836 MVA & 1372 MVA & BI & $\mathrm{AB}, 15 \%, 2 \Omega$ \\
\hline 11 & 0 MVA & 3000 MVA & BI & ABCG, $15 \%, 2 \Omega$ \\
\hline 12 & 1836 MVA & 1372 MVA & BI & $\mathrm{ABCG}, 15 \%, 2 \Omega$ \\
\hline
\end{tabular}

Table 4 Settings of the studied physical relay

\begin{tabular}{|c|c|c|}
\hline Parameters Name & \multicolumn{2}{|c|}{ Distance Relay Settings } \\
\hline Characteristic & Memory-polarised MHO & QUAD \\
\hline Polarising Factor, $p$ & $p=1$ & NA \\
\hline Reach Setting, $Z_{r}$ & Zone & \\
\hline Residual Compensation Factor, $K_{0}$ & & \\
\hline Time Delay, $t_{d e}$ & Zone 1 & \\
\hline Right Resistive Reach & N/A & $6.72 \Omega$ \\
\hline Left Resistive Reach & N/A & $1.68 \Omega$ \\
\hline Directional Angle & N/A & $30^{\circ}$ \\
\hline Tilt Angle & N/A & $-3^{\circ}$ \\
\hline
\end{tabular}

Table 5 HIL testing results of the studied physical relay

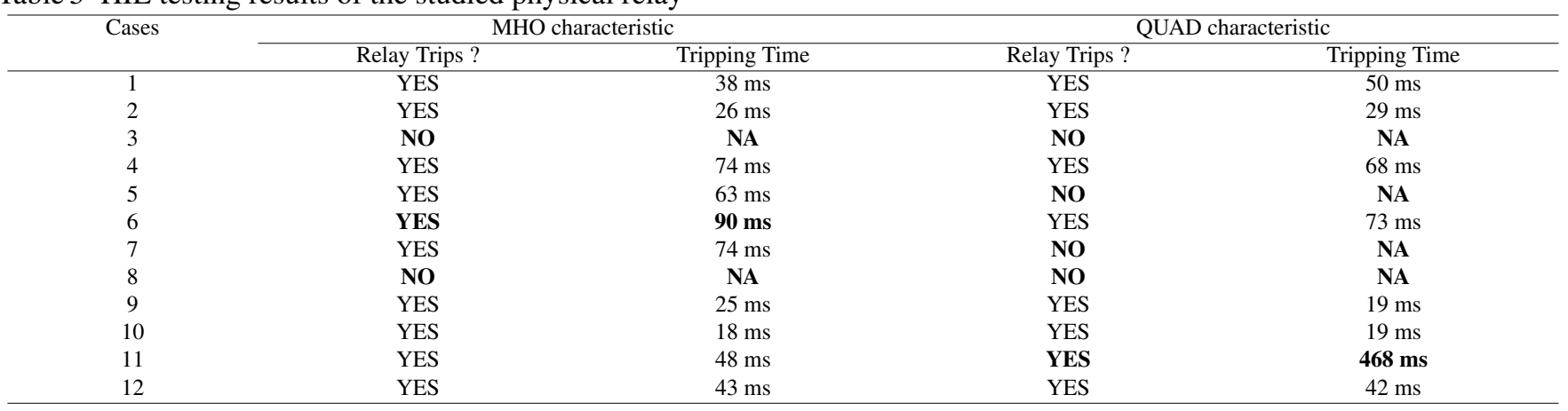

\subsection{Protection settings of physical relay}

In this paper, two typically used protection characteristics, i.e. MHO and QUAD, with the settings presented in Table 4 are employed in the distance relay. For the MHO characteristic, the memory polarising technique is applied to address the reliability issues in the case of the close-up faults, where vectors, $S_{1}$ and $S_{2}$, used in phase comparator are depicted as (7) and (8) [16].

$$
\begin{gathered}
S_{1}=V+p V_{m e m} \\
S_{2}=V-I Z_{R}
\end{gathered}
$$

where $p$ is the memory polarising factor, which is set as 1 in this test; $V_{m e m}$ is the memory polarised voltage, which is typically the phase voltage several cycles before the fault; $Z_{R}$ is the relay setting impedance. For the relay with the memorypolarised $\mathrm{MHO}$ characteristic, it will consider the fault being within the protection zone if the angle between vector $S_{1}$ and $S_{2}$, marked as $\theta$, is larger than $90^{\circ}$ as indicated in (9).

$$
\theta=\operatorname{ang}\left(\frac{S_{1}}{S_{2}}\right)>90^{\circ}
$$

\subsection{Test results of distance relay with $\mathrm{MHO}$ based relay}

The testing results are shown in Table 5. Based on the Grid Code [9], the faults in the transmission network with voltage level being greater than $200 \mathrm{kV}$ should be isolated no more than $140 \mathrm{~ms}$. Assuming a circuit breaker (CB) operating time to be approximately $50 \mathrm{~ms}$, thus the maximum allowed operating time of relay is selected as $90 \mathrm{~ms}$ in this study. In practice, some other delays will be introduced in the fault isolation process and in some cases, the operating time of $\mathrm{CB}$ will be longer than $50 \mathrm{~ms}$, therefore, the required time of $90 \mathrm{~ms}$ is intentionally set at the high end, so that any operating time observed in the tests longer than this value will indeed indicate compromised performance that is not acceptable for transmission system protection. The cases with protection issues of $\mathrm{MHO}$ relay are analysed in detail from Section 4.3.1 to 4.3.3.

4.3.1 Analysis of issues observed in Case 3: In case 3, the relay does not trip as it should. The value of angle $\theta$ of the investigated relay is plotted in Fig. 3, which determines the tripping action of the relays as discussed in Section 4.2. As the internal functional blocks and signals of the physical relay are largely inaccessible, the plotted angles $\theta$ in the following cases are from an analysing tool developed the RSCAD software by the authors based on the relay manual. Therefore, there could be some differences between the actual $\theta$ in the physical relay and the presented angle $\theta$. Based on the results shown in Fig. 3, the relay should trip as the value of $\theta$ is larger than $90^{\circ}$. From a further investigation, it was found that the failed 


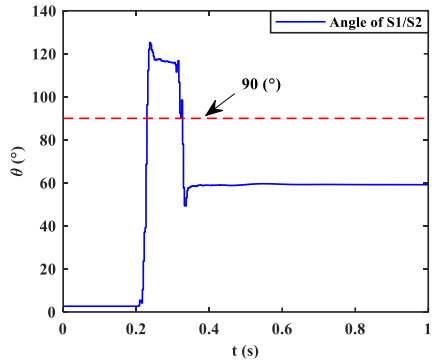

Fig. 3. Angle $\theta$ of case 3
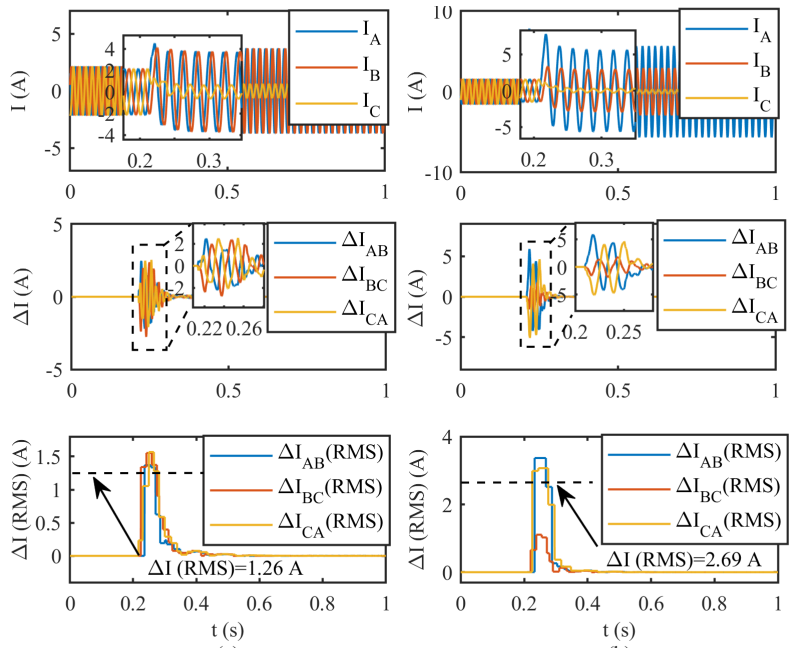

Fig. 4 Phase currents and calculated superimposed currents measured by relay, (a) case 3, (b) case 6 .

relay operation is due to the conflict between phase selection and impedance measurement units. For the studied relay, the faulted phase is selected by the magnitudes of the three phaseto-phase superimposed currents, i.e., $\Delta I_{A B}, \Delta I_{B C}$ and $\Delta I_{C A}$, and any superimposed current greater than $80 \%$ of the largest superimposed current is included in the phase selection logic. For the investigated AG fault, the relay will detect this fault if the superimposed currents, $\Delta I_{A B}$ and $\Delta I_{C A}$, both increase to a similar value and the value of $\Delta I_{B C}$ closes to zero. However, by observing the superimposed currents as shown in Fig. 4 (a), all of those three superimposed currents are larger than the current threshold of phase selection logic, i.e., RMS value of $1.26 \mathrm{~A}$ ( $80 \%$ of the largest superimposed current). Such phenomenon is caused by the converter's control during fault, and as a result, the conflict between the impedance measuring element and phase selection element blocked the tripping action.

4.3.2 Analysis of issues observed in case 6: From the results in Table 5, the studied relay fails to trip in case 3 . Therefore, in case 6, the SG1 with the fault level of 839 MVA is connected at the HVDC side to increase the local system fault level to assist the operation of distance relays. Based on the results in Fig. 4 (b), after the connection of SG1, the value of $\Delta I_{A B}$ and $\Delta I_{C A}$ are both greater than the current threshold, i.e., 2.69 A (RMS value). Therefore, the AG fault can be detected by the phase selection unit of the relay. In this condition, distance relay trips after $90 \mathrm{~ms}$ of the fault.

4.3.3 Analysis of issues observed in case 8: In case 8, the studied relay fails to trip in a bolted $A B$ fault when the HVDC employs the constant reactive power control, which is illustrated in Fig. 5. From Fig. 5 (a), it is clear that the currents in phase A and B are almost in phase and with the same magnitude during the $\mathrm{AB}$ fault. Therefore, the value of the denominator in (10) will be close to zero, which causes the numerical instability of the impedance measurement as shown in Fig. 5 (b). This instability can also be reflected by the measured angle $\theta$ as displayed in Fig. 6, where the values of the angle $\theta$ experience a significant oscillation during the fault period. In this case, the relay fails to trip. More information about this issue can be found in [7].

$$
Z_{A B}=\frac{V_{A}-V_{B}}{I_{A}-I_{B}}
$$

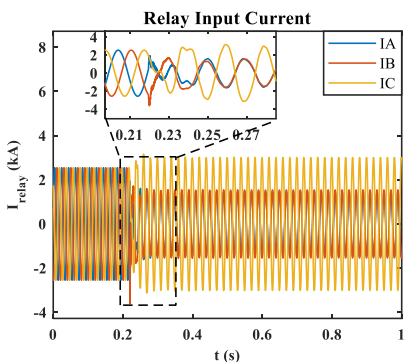

(a)

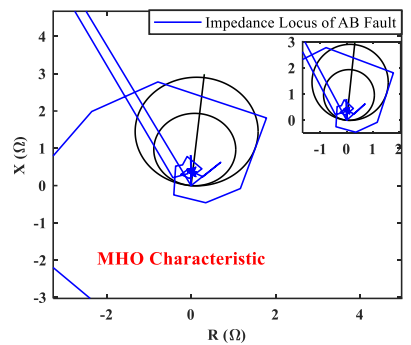

(b)
Fig. 5 Results of case 8, (a) input currents, (b) impedance locus.

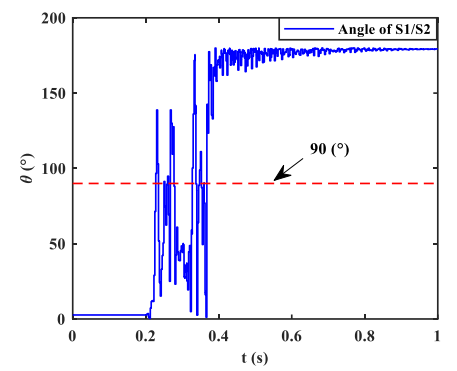

Fig. 6. Angle $\theta$ of Case 8.

\subsection{Test results of distance relay with QUAD characteristic}

The HIL test results of QUAD based relay are displayed in Table 5. Based on the investigation, some observed issues are caused by the same reasons as analysed in the Section 4.3 (e.g., the phase selection issue in case 3 , the impedance measuring issues in case 8). Therefore, to avoid repetition, only the results of the case 5, case 7 and case 11 will be analysed in this section.

4.4.1 Analysis of issues observed in case 5: In case 5, the relay fails to trip the AG fault when the HVDC uses the constant reactive power control. Presently, it is unclear the root cause for the failed operation due to the lack of information of the internal logics of the relay. This represents a part for future research involving engagement with the manufacturers.

4.4.2 Analysis of issues observed in case 7: In case 7, the relay does not trip during the AB fault when the HVDC employs the balanced current control strategy. To further investigate the source of this issue, the angle difference of the currents from both sides has been displayed in Fig. 7 (a). From the figure, the value of the $\Delta \psi$ equals to $-82.63^{\circ}$ and in the studied case, the fault level of remote-end network is almost three times of the local HVDC system. Based on the analysis in the Section 3.2, 
in this condition, the measured reactance of the distance relay will experience a severe error, which results the impedance locus appears at the reverse side of the protective zone as shown in Fig. 7 (b). This clearly shows that the introduction of the converter-based sources with current characteristic different from conventional voltage source can lead to severe impedance measurement issues.
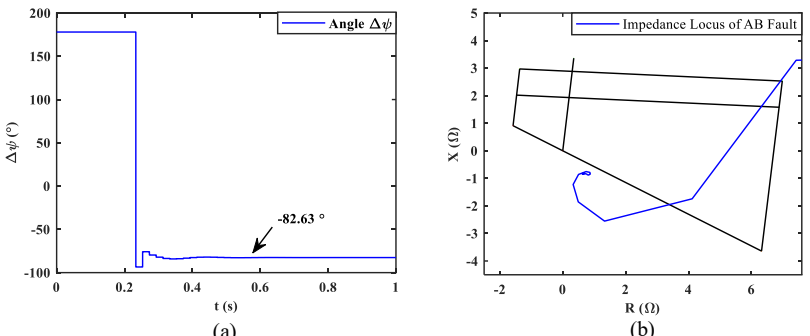

Fig. 7. Results of case 7, (a) angle $\Delta \psi$, (b) impedance locus.
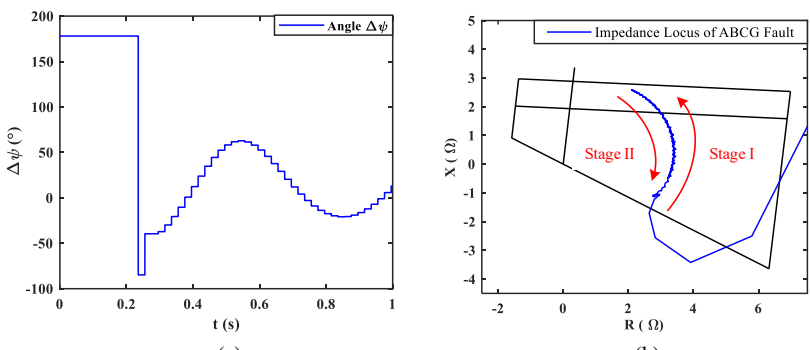

Fig. 8. Results of case 11, (a) angle $\Delta \psi$, (b) impedance locus

4.4.3 Analysis of issues observed in case 11: In case 11, an ABCG fault is applied in the protected line. Based on the results in Table 5 , the relay suffers from the zone discrimination issue, i.e. it trips in zone 2 for the fault at $15 \%$ of the line. Given that the three-phase voltage of network drops to almost zero in the case of ABCG faults, the phase-locked loop (PLL) of the HVDC system cannot track system voltage effectively, which causes the severe oscillation of $\Delta \psi$ as shown in Fig. 8 (a). As stated in Section 3.2, the varying $\Delta \psi$ will further introduce the increase (positive $\Delta \psi$ ) or the decrease (negative $\Delta \psi$ ) of the measured impedance of distance relay as shown in Fig. 8 (b), which results in the zone discrimination issues of the relay.

\section{Conclusions}

In this paper, comprised distance protection performance (e.g., failed tripping, delayed tripping and zone discrimination issues) with the integration of HVDC systems (and converters in general) has been identified via the HIL tests of one physical relay. To further understand the sources of the observed protection issues, theoretical analysis was conducted. Based on the results, it can be concluded that the connection of HVDC system can, 1) introduce the under/over reach impedance measuring issues owing to the uncertain angle of the currents injected by the HVDC system; 2) unstable impedance measuring issues caused by the identical current phases of the two faulted phases in the case of $\mathrm{AB}$ faults when the HVDC employs the constant reactive current control; and 3) faulted phase selection issue owing to the different fault current characteristics of converters from SGs. Furthermore, different protection characteristics (MHO and QUAD) are also considered in this paper, and it was found that owing to the dynamic expansion of the protective zone of the memory-polarised MHO, the relay with MHO characteristic could have a better performance than the QUAD based distance relay in the events of faults locating close to the relay and driven mainly by converters from the relay side.

\section{Acknowledgements}

This work is sponsored by the National HVDC Centre in the UK.

\section{References}

[1] National Grid ESO, Future Energy Scenarios, 2020.

[2] R. Li et al., "Impact of low (zero) carbon power systems on power system protection: a new evaluation approach based on a flexible modelling and hardware testing platform," in IET Renewable Power Generation, vol. 14, no. 5, pp. 906-913, 2020.

[3] B. J. Brearley and R. R. Prabu, "A review on issues and approaches for microgrid protection," Renewable and Sustainable Energy Reviews, vol. 67, pp. 988-997, 2017.

[4] D. Liu et al., "Impact of System Strength and HVDC Control Strategies on Distance Protection Performance," 9th International Conference on Renewable Power Generation, Dublin, Ireland, 2021.

[5] A. Hooshyar et al., "Distance Protection of Lines Emanating From Full-Scale Converter-Interfaced Renewable Energy Power Plants-Part I: Problem Statement," in IEEE Trans. on Power Delivery, vol. 30, no. 4, pp. 1770-1780, Aug. 2015.

[6] S. Paladhi et al., "Adaptive Distance Protection for Lines Connecting Converter-Interfaced Renewable Plants," in IEEE Journal of Emerging and Selected Topics in Power Electronics, 2020.

[7] J. Jia et al., "Impact of VSC Control Strategies and Incorporation of Synchronous Condensers on Distance Protection Under Unbalanced Faults," in IEEE Trans. on Industrial Electronics, vol. 66, no. 2, pp. 1108-1118, Feb. 2019.

[8] P. Rodriguez et al., "Safe current injection strategies for a STATCOM under asymmetrical grid faults," 2010 IEEE Energy Conversion Congress and Exposition, Atlanta, GA, 2010, pp. 3929-3935.

[9] The Grid Code Issue 5 Revision 21, National Grid, London, UK, May 2020.

[10] 'Evaluation of HVDC with Synchronous Condenser impact on AC Protection - The National HVDC Centre', https://www.hvdccentre.com/innovation/dc_with_ac_protection.

[11] RTDS Technology Inc., "Real Time Digital Simulator Power System Components Manual".

[12] A. Banaiemoqadam et al., "A Control-Based Solution for Distance Protection of Lines Connected to Converter-Interfaced Sources During Asymmetrical Faults," in IEEE Trans. on Power Delivery, vol. 35, no. 3, pp. 1455-1466, June 2020.

[13] Y. Fang, K. Jia, Z. Yang, Y. Li and T. Bi, "Impact of InverterInterfaced Renewable Energy Generators on Distance Protection and an Improved Scheme," in IEEE Trans. on Industrial Electronics, vol. 66, no. 9, pp. 7078-7088, Sept. 2019.

[14] M. Rahman, et al., Development and Validation of Offline and Real-Time User-Defined Models of Alternative MMC Configurations, April 2018.

[15] H. Song et al., "Dual current control scheme for PWM converter under unbalanced input voltage conditions," in IEEE Trans. on Industrial Electronics, vol. 46, no. 5, pp. 953-959, Oct. 1999.

[16] Technical Manual: MiCOM, P40 Agile P443, June 2020. 